\title{
Historia familiar de enfermedad tiroidea y riesgo de hipotiroidismo congénito en neonatos con síndrome de Down
}

\author{
Jorge R. Corona-Rivera, ${ }^{1,2 *}$ Topacio O. Andrade-Romo, ${ }^{1}$ Liuba M. Aguirre-Salas, ${ }^{3}$ Lucina Bobadilla-Morales, ${ }^{1,2}$ \\ Cristian I. Aranda-Sánchez, ${ }^{1}$ Alfredo Corona-Rivera, ${ }^{1,2}$ y René O. Pérez-Ramírez ${ }^{4}$ \\ ${ }^{1}$ Servicio de Genética y Unidad de Citogenética, Centro de Registro e Investigación sobre Anomalías Congénitas, Hospital Civil de Guadalajara \\ Dr. Juan I. Menchaca; ${ }^{2}$ Departamento de Biología Molecular y Genómica, Instituto de Genética Humana Dr. Enrique Corona-Rivera, Universidad \\ de Guadalajara; ${ }^{3}$ Servicio de Endocrinología, Hospital Civil de Guadalajara Dr. Juan I. Menchaca; ${ }^{4}$ Servicio de Neonatología, Hospital Civil de \\ Guadalajara Dr. Juan I. Menchaca. Guadalajara, Jal., México
}

\section{Resumen}

Introducción: La historia familiar de enfermedad tiroidea (HFET) como factor de riesgo para hipotiroidismo congénito (HC), en síndrome de Down (SD) aún no ha sido explorada. Objetivo: Determinar si la HFET está asociada a mayor riesgo de HC en neonatos con SD. Método: Estudio de casos y controles en 220 neonatos con SD. Se compararon las pruebas de función tiroidea (PFT) de 37 con SD e HFET (casos), frente a las PFT de 183 recién nacidos con SD sin HFET (grupo de referencia). Se realizó análisis de regresión logística multivariante y se calculó la razón de momios (RM) y sus respectivos intervalos de confianza del 95 \% (IC 95 \%). Resultados: Nueve casos HC (4.1\%). El HC mostró asociación con la HFET (RMa = 8.3, IC $95 \%$ : 2.0-34.3), particularmente en los varones (RMa = 9.0, IC $95 \%$ : 1.6-49.6). La ausencia de HFET tuvo una RM de protección para HC (RMa = 0.4, IC $95 \%$ : 0.1-0.8). Conclusiones: La HFET puede es una estrategia fácil y accesible para identificar pacientes con $S D$ con mayor riesgo de $H C$.

PALABRAS CLAVE: Síndrome de Down. Hipotiroidismo congénito. TSH. T4 libre. Historia familiar de enfermedad tiroidea.

\section{Family history of thyroid disease and risk of congenital hypothyroidism in neonates with Down syndrome}

\begin{abstract}
Introduction: Family history of thyroid disease (FHTD) as risk factor for congenital hypothyroidism (CH) in patients with Down syndrome (DS) has not yet been explored. Objective: To determine whether FHTD is associated with an increased risk for CH in DS. Method: Case-control study in 220 neonates with DS. Thyroid function tests of 37 infants with DS and FHTD (cases) were compared with those of 183 DS newborns without FHTD (reference group). Data were analyzed using multivariate logistic regression analysis and adjusted odds ratios (aORs) with their respective $95 \%$ confidence intervals (Cl) were calculated. Results: Nine newborns with DS in our sample had CH (4.1\%). FHTD showed an association with $\mathrm{CH}$ in neonates with $D S(a O R=8.3,95 \% \mathrm{Cl}: 2.0-34.3)$, particularly in males $(a O R=9.0,95 \% \mathrm{Cl}$ : 1.6-49.6). In contrast, newborns with DS without FHTD were less likely to suffer from $\mathrm{CH}(a O R=0.4,95 \% \mathrm{Cl}$ : 0.1-0.8). Conclusions: FHTD detailed evaluation can be an easy and accessible strategy to identify those newborns with $\mathrm{DS}$ at higher risk for $\mathrm{CH}$.
\end{abstract}

KEY WORDS: Down syndrome. Congenital hypothyroidism. TSH. Free T4. Family history of thyroid disease. 


\section{Introducción}

Los pacientes con síndrome de Down (SD) tienen un riesgo aumentado de hipotiroidismo congénito (HC). Las pruebas para detectar el $\mathrm{HC}$ al nacimiento están recomendadas para todos los recién nacidos (RN) con SD. En programas de tamizaje, el 1-2 \% de los RN con SD son detectados con $\mathrm{HC}$, lo que equivale a 1 de cada $141 \mathrm{RN}$ con SD, proporción 28 veces mayor que la observada en la población general. ${ }^{2-4}$ Además del $\mathrm{HC}$, las pruebas de función tiroidea (PFT) frecuentemente detectan también hipotiroidismo subclínico y/o cambios en los niveles plasmáticos de hormona estimulante tiroidea (TSH) y tiroxina (T4), todos de origen tiroideo. ${ }^{5}$ Se ha sugerido que la trisomía 21 causa desequilibrios en genes sensibles a dosis (como IFNAR1 e IFNAR2), interfiriendo con la producción de hormona tiroidea o en la ontogénesis tiroidea. ${ }^{6}$ No obstante, pueden estar involucrados otros factores de susceptibilidad, aparte de la trisomía 21.

La autoinmunidad está fuertemente implicada en la ocurrencia de la tiroiditis de Hashimoto, o la enfermedad de Graves en los niños mayores y adultos con SD, ${ }^{4,7}$ pero no en el $\mathrm{HC}$, que se considera un trastorno no autoinmune. ${ }^{5,6}$ Hace más de 50 años, Fialkow et al..$^{8}$ observaron un aumento en la frecuencia de anticuerpos tiroideos en madres de neonatos con SD, evidenciando una clara asociación entre SD y enfermedad tiroidea en los RN, sin embargo, no estudiaron la función tiroidea neonatal. La historia familiar de enfermedad tiroidea (HFET) se ha descrito como factor de riesgo para $\mathrm{HC}$ en niños. ${ }^{9} \mathrm{Sin}$ embargo, la posible relación de la HFET con el HC en el SD aún no ha sido explorada. En un estudio previo, ${ }^{10}$ observamos que la HFET fue 2.3 veces mayor entre los $\mathrm{RN}$ con SD con respecto a un grupo control de RN no malformados. El objetivo del presente trabajo fue investigar si la HFET incrementa el riesgo de $\mathrm{HC}$ entre los $\mathrm{RN}$ con SD comparado con el grupo de referencia de RN con SD sin HFET.

\section{Método}

De enero de 2009 a diciembre del 2018, realizamos un estudio de casos y controles en $220 \mathrm{RN}$ con SD, incluyendo $37 \mathrm{RN}$ con SD e HFET (casos) y $183 \mathrm{RN}$ con SD sin HFET (grupo de referencia), todos nacidos en el Hospital Civil de Guadalajara Dr. Juan I. Menchaca (Guadalajara, Jalisco, México). Los datos fueron recolectados por el Centro de Registro e Investigación de Anomalías Congénitas (CRIAC), nuestro programa hospitalario de vigilancia de defectos congénitos, que incluye a todos los $\mathrm{RN}$ vivos de $\geq 20$ semanas de gestación y peso $\geq 500 \mathrm{~g}$. Solo se incluyeron pacientes con diagnóstico de trisomía 21 confirmada mediante cariotipo de sangre periférica realizado en nuestra unidad de citogenética y que además tuvieran mediciones de TSH y T4 realizadas durante el periodo neonatal. Se excluyeron a aquellos pacientes que no contaron con PFT o estas fueron incompletas. El estudio fue aprobado por el Comité de Ética del Hospital Civil de Guadalajara Juan I. Menchaca y se siguieron los estándares éticos establecidos en la declaración de Helsinki de 1964 y sus posteriores enmiendas. Se contó con la aprobación de la Junta de Ética en Investigación de la Secretaría de Salud de Jalisco (\#0116/16 HCJM/2016).

Las determinaciones de TSH y T4 fueron realizadas a una edad media de 10.9 días al momento del estudio (66 \% entre el tercer y décimo día). La T4 fue medida en forma de T4 libre, T4 total o ambas, dependiendo de la disponibilidad del laboratorio de nuestro hospital. Ninguno de nuestros pacientes había recibido tratamiento para hipotiroidismo antes de la obtención de las muestra para las PFT. Las concentraciones de TSH, T4 libre y T4 total fueron determinadas de acuerdo con los estándares de procedimientos operativos en nuestro laboratorio central. Todas las muestras se analizaron en un sistema de inmunoensayo UniCel ${ }^{\mathrm{TM}} \mathrm{Dxl} 800$ con acceso automatizado (Beckman Coulter Inc., Brea, CA), empleando un sistema de detección de quimioluminiscencia directa conforme a las instrucciones del fabricante.

Los resultados de las PFT fueron interpretados empleando intervalos estandarizados de referencia específicos para la edad al momento de la toma.11,12 Los resultados de las PFT fueron clasificados de acuerdo con Jonklaas et al..$^{13}$ en: 1) HC primario, TSH elevada con T4 libre y/o T4 total bajas con respecto a los intervalos de referencia; 2) hipertirotropinemia, TSH alta con T4 libre y/o T4 total dentro de los intervalos de referencia; 3 ) síndrome de tiroides enferma, TSH baja y T4 libre y/o T4 total, todas menores al intervalo de referencia, y 4) eutiroideo, TSH, T4 libre y T4 total normales.

El registro usado por el CRIAC se obtuvo mediante entrevista a las madres de los bebés con SD poco después del nacimiento, previo consentimiento informado por escrito para participar en el estudio y permitir la revisión del expediente médico. En todos los pacientes se obtuvo la historia familiar mediante el método de árbol genealógico de tres generaciones. ${ }^{14}$ Los RN 
con SD provenientes de familias con uno o más miembros afectados por cualquier enfermedad tiroidea constituyeron el grupo de casos y aquellos con HFET negativa, el grupo de referencia. La historia familiar recopilada incluyó preguntas específicas y detalles con respecto a la presencia y tipo de enfermedad tiroidea entre los familiares del caso índice. También fueron evaluados otros potenciales factores de riesgo $\mathrm{HC}^{15}$ incluyendo: edad materna $\geq 35$ años, paridad $\geq 4$ y presencia de diabetes durante el embarazo, así como el autorreporte de consumo de tabaco y alcohol durante la gestación. También se registró la vía de nacimiento, el sexo del recién nacido, edad gestacional, peso al nacimiento $(<2,000 \mathrm{~g})$, puntuación de Apgar $<7$ a los 5 min y la presencia de defectos congénitos cardiacos y/o malformaciones gastrointestinales.

Los valores promedio \pm error estándar de la media de TSH, T4 libre y T4 total fueron comparados usando la prueba de $U$ de Mann-Whitney, ya que no seguían una distribución normal según la prueba de KolmogorovSmirnov. Los resultados de las características maternas y de las variables en los RN fueron comparados entre los RN con SD y aquellos del grupo de referencia mediante la prueba de $\chi^{2}$; se consideró un valor de $p<0.05$ como estadísticamente significativo. Los factores que mostraron diferencias estadísticas entre los grupos de estudio fueron incluidos en un análisis de regresión logística multivariante para evaluar la desviación explicada por las diferentes variables de interés, usando el método de enter y expresada como RM ajustada (RMa) con sus respectivos intervalos de confianza del $95 \%$ (IC $95 \%$ ). El análisis de los datos se realizó empleando el programa SPSS (IBM SPSS Statistics, Versión 21.0; IBM Corp.; Armonk, NY, EE.UU.).

\section{Resultados}

De un total de 269 pacientes nacidos con SD en nuestro hospital durante el periodo de estudio, 220 (81.8\%) cumplieron los criterios de inclusión (206 con trisomía 21 completa, 8 con trisomía 21 mosaico y 6 con trisomía por translocación), no incluyendo a 49 que tuvieron PFT incompletas y/o su confirmación por cariotipo. Treinta y siete pacientes (16.8\%) con SD e HFET (grupo de casos) fueron comparados con 183 pacientes con SD (83.2 \%) e HFET negativa (grupo de referencia), 126 del sexo masculino (57.3\%) y 94 del sexo femenino (42.7\%), con una proporción masculino:femenino de 1.3:1.

La Tabla 1 muestra la HFET obtenida en las 37 familias del grupo de casos. Solo siete casos índice
Tabla 1. Historia de enfermedad tiroidea en 37 familias de neonatos con síndrome de Down

\begin{tabular}{|l|c|}
\hline Historia familiar de enfermedad tiroideas & $\begin{array}{c}\text { Número de } \\
\text { familias }\end{array}$ \\
\hline Madre con hipotiroidismo & 4 \\
\hline Hermana con hipotiroidismo & 3 \\
\hline Al menos un familiar afectado de segundo grado & 19 \\
\hline Al menos un familiar afectado de tercer grado & 18 \\
\hline Al menos un familiar afectado en el lado materno & 25 \\
\hline Al menos un familiar afectado en el lado paterno & 11 \\
\hline Al menos un familiar afectado en la familia & 4 \\
\hline $\begin{array}{l}\text { Tipo de enfermedad tiroidea en la familia } \\
\text { Hipotiroidismo }\end{array}$ & 30 \\
\hline Hipertiroidismo \\
$\begin{array}{l}\text { Bocio } \\
\text { Enfermedad tiroidea no especificada }\end{array}$ \\
\hline $\begin{array}{l}\text { Número de familiares afectados } \\
\text { Uno } \\
\text { Dos } \\
\text { Tres o más }\end{array}$ & 2 \\
\hline
\end{tabular}

tuvieron historia de un familiar de primer grado con enfermedad tiroidea (madre o hermana) y en la mayoría de familias, el afectado referido tenía una relación de parentesco de segundo o tercer grado. En cada caso índice, el número de familiares afectados con enfermedad tiroidea fue de uno, dos y tres o más en 24,11 y 3 , respectivamente. El hipotiroidismo $(n=30)$ fue la enfermedad tiroidea más frecuentemente referida en la mayoría de estas familias, pero también incluyeron hipertiroidismo $(n=4)$, bocio $(n=2)$ y enfermedad tiroidea no especificada $(n=4)$.

En la Tabla 2 se muestran los potenciales factores de riesgo para $\mathrm{HC}$ en relación con la presencia de la HFET en la muestra estudiada. Hubo mayor número de afectados del sexo femenino en el grupo de casos en comparación con el grupo de referencia $(R M=2.3$, IC $95 \%$ : 1.1-4.6, $p=0.029$ ), mientras que en el grupo de referencia predominaron los afectados del sexo masculino (RM = 0.4, IC $95 \%$ : 0.2-0.9, $p=0.029$ ). Para el resto de las características evaluadas entre los grupos de estudio, no se encontraron otras diferencias estadísticas en el análisis bivariante.

La Tabla 3 muestra el promedio de los valores de las PFT comparados con la HFET. La media de T4 libre fue menor en el grupo de los casos que en el grupo de referencia $(p=0.004)$. No se encontraron otras diferencias significativas. Los diagnósticos tiroideos de acuerdo con la presencia de HFET y sus RM 
Tabla 2. Características maternas y resultados del embarazo en relación con la historia familiar de enfermedad tiroidea entre los neonatos con síndrome de Down de la muestra estudiada

\begin{tabular}{|c|c|c|c|c|}
\hline \multirow[t]{3}{*}{ Variables } & \multicolumn{2}{|c|}{ Historia familiar de enfermedad tiroidea } & \multirow[t]{3}{*}{$p^{*}$} & \multirow[t]{3}{*}{ RM (IC $95 \%)$} \\
\hline & Presente & Ausente & & \\
\hline & $n=37(\%)$ & $n=183(\%)$ & & \\
\hline $\begin{array}{l}\text { Historia familiar } \\
\text { Consanguinidad } \\
\text { Familiares con cardiopatía congénita } \\
\text { Familiares con síndrome de Down }\end{array}$ & $\begin{array}{l}1(2.7) \\
4(10.8) \\
9(24.3)\end{array}$ & $\begin{array}{c}4(2.2) \\
14(7.6) \\
41(22.4)\end{array}$ & $\begin{array}{l}1.000 \\
0.514 \\
0.831\end{array}$ & $\begin{array}{l}1.2(0.1-11.4) \\
1.5(0.4-4.7) \\
1.1(0.5-2.5)\end{array}$ \\
\hline $\begin{array}{l}\text { Características maternas } \\
\text { Edad materna (años, media } \pm \text { DE) } \\
\leq 19 \text { años } \\
\geq 35 \text { años }\end{array}$ & $\begin{array}{c}29.5 \pm 8.7 \\
6(16.2) \\
11(29.7)\end{array}$ & $\begin{array}{l}29.9 \pm 9.2 \\
36(19.7) \\
71(38.8)\end{array}$ & $\begin{array}{l}0.785^{\dagger} \\
0.819 \\
0.354\end{array}$ & $\begin{array}{l}0.8(0.3-2.0) \\
0.7(0.3-1.4)\end{array}$ \\
\hline Nivel socioeconómico bajo & $8(21.6)$ & $50(27.3)$ & 0.544 & $0.7(0.3-1.7)$ \\
\hline Nulípara & $9(24.3)$ & $52(28.4)$ & 0.691 & $0.8(0.4-1.8)$ \\
\hline Paridad $\geq 4$ & $15(40.5)$ & $68(37.1)$ & 0.713 & $1.1(0.6-2.4)$ \\
\hline $\begin{array}{l}\text { IMC pregestacional }\left(\mathrm{kg} / \mathrm{m}^{2}, \text { media } \pm \mathrm{DE}\right)^{\ddagger} \\
\quad<18.5 \mathrm{~kg} / \mathrm{m}^{2} \\
\geq 25 \mathrm{~kg} / \mathrm{m}^{2} \\
\geq 30 \mathrm{~kg} / \mathrm{m}^{2}\end{array}$ & $\begin{array}{l}26.1 \pm 4.7 \\
1(2.8) \\
18(51.4) \\
7(20.0)\end{array}$ & $\begin{array}{c}25.7 \pm 5.1 \\
5(3.3) \\
70(45.8) \\
32(20.9)\end{array}$ & $\begin{array}{l}0.674^{\dagger} \\
1.000 \\
0.577 \\
1.000\end{array}$ & $\begin{array}{l}0.9(0.1-7.7) \\
1.2(0.6-2.6) \\
0.9(0.4-2.4)\end{array}$ \\
\hline $\begin{array}{l}\text { Ganancia de peso gestacional }(\mathrm{kg}, \text { media } \pm \mathrm{DE})^{\ddagger} \\
\text { Insuficiente } \\
\text { Adecuado } \\
\text { Excesivo }\end{array}$ & $\begin{array}{l}8.7 \pm 5.5 \\
20(57.1) \\
8(22.8) \\
7(20.0)\end{array}$ & $\begin{array}{l}9.0 \pm 6.8 \\
67(43.8) \\
61(39.9) \\
25(16.3)\end{array}$ & $\begin{array}{l}0.778^{\dagger} \\
0.190 \\
0.079^{\ddagger} \\
0.622\end{array}$ & $\begin{array}{l}1.7(0.8-3.5) \\
0.4(0.2-1.0) \\
1.3(0.5-3.2)\end{array}$ \\
\hline Diabetes gestacional & $1(2.7)$ & $14(7.7)$ & 0.476 & $0.3(0.0-2.6)$ \\
\hline Consumo de tabaco durante el embarazo & $7(18.9)$ & $26(14.2)$ & 0.455 & $1.4(0.6-3.5)$ \\
\hline Consumo de alcohol durante el embarazo & $6(16.2)$ & $26(14.2)$ & 0.798 & $1.2(0.4-3.1)$ \\
\hline Nacimiento vía vaginal & $13(35.1)$ & $78(42.6)$ & 0.466 & $0.7(0.3-1.5)$ \\
\hline Nacimiento vía cesárea & $22(59.4)$ & $100(54.6)$ & 0.717 & $1.2(0.6-2.5)$ \\
\hline $\begin{array}{l}\text { Recién nacidos } \\
\text { Femenino } \\
\text { Masculino }\end{array}$ & $\begin{array}{l}22(59.5) \\
15(40.5)\end{array}$ & $\begin{array}{l}72(39.3) \\
111(60.7)\end{array}$ & $\begin{array}{l}0.029 \\
0.029\end{array}$ & $\begin{array}{l}2.3(1.1-4.6) \\
0.4(0.2-0.9)\end{array}$ \\
\hline $\begin{array}{l}\text { Edad gestacional (semanas, media } \pm \mathrm{DE} \text { ) } \\
\text { Nacimiento pretérmino }\end{array}$ & $\begin{array}{c}37.4 \pm 2.2 \\
10(27.0)\end{array}$ & $\begin{array}{l}37.5 \pm 1.9 \\
50(27.3)\end{array}$ & $\begin{array}{c}0.740^{+} \\
1.000\end{array}$ & $0.9(0.4-2.2)$ \\
\hline Talla $(\mathrm{cm}$, media $\pm \mathrm{DE})$ & $46.7 \pm 3.9$ & $46.8 \pm 3.0$ & $0.744^{\dagger}$ & \\
\hline $\begin{array}{l}\text { Peso ( } \mathrm{g}, \text { media } \pm \mathrm{DE}) \\
\text { Pequeño para edad gestacional } \\
\text { Peso }<2,000 \mathrm{~g}\end{array}$ & $\begin{array}{l}2501 \pm 623 \\
9(24.3) \\
7(18.9)\end{array}$ & $\begin{array}{l}2591 \pm 619 \\
37(20.2) \\
37(20.2)\end{array}$ & $\begin{array}{l}0.421^{\dagger} \\
0.658 \\
1.000\end{array}$ & $\begin{array}{l}1.3(0.5-2.9) \\
0.9(0.4-2.2)\end{array}$ \\
\hline Perímetro cefálico (cm, media $\pm \mathrm{DE})$ & $31.6 \pm 2.0$ & $31.7 \pm 3.6$ & $0.817^{\dagger}$ & \\
\hline Apgar < 7 en el min 1 & $6(16.2)$ & $39(21.3)$ & 0.655 & $0.7(0.3-1.8)$ \\
\hline Apgar $<7$ en el $\min 5$ & $2(5.4)$ & $12(6.6)$ & 1.000 & $0.8(0.2-3.8)$ \\
\hline Cardiopatía congénita & $12(32.4)$ & $72(39.3)$ & 0.464 & $0.7(0.3-1.6)$ \\
\hline Malformación gastrointestinal & $2(5.4)$ & $15(8.2)$ & 0.744 & $0.6(0.1-2.9)$ \\
\hline
\end{tabular}

*Resultado del análisis con $\chi^{2}$ de ambas columnas.

tPrueba t de Student.

fDisponible en 35 y 153 madres del grupo de casos y el grupo referencia, respectivamente.

$\mathrm{RM}$ = razón de momios; IC 95 \% = intervalo de confianza 95 \%; DE = desviación estándar. 
Tabla 3. Pruebas de función tiroidea en neonatos con síndrome de Down de acuerdo con la historia familiar de enfermedad tiroidea

\begin{tabular}{|c|c|c|c|c|c|}
\hline \multirow[t]{3}{*}{ Variables } & \multicolumn{4}{|c|}{ Historia familiar de enfermedad tiroidea } & \multirow[t]{3}{*}{$\mathbf{p}^{*}$} \\
\hline & \multicolumn{2}{|c|}{ Positiva } & \multicolumn{2}{|c|}{ Negativa } & \\
\hline & $n$ & Media \pm DE (rango) & n & Media \pm DE (rango) & \\
\hline Edad al diagnóstico (días) & 37 & $12.3 \pm 8.1(4-30)$ & 183 & $10.6 \pm 7.9(3-30)$ & 0.241 \\
\hline $\mathrm{TSH}(\mu \mathrm{U} / \mathrm{ml})$ & 37 & $10.99 \pm 16.3(1.2-95.4)$ & 183 & $6.82 \pm 6.7(0.2-54.3)$ & 0.011 \\
\hline T4I (ng/dl) & 26 & $1.11 \pm 0.4(0.5-2.1)$ & 115 & $1.54 \pm 1.1(0.5-11.6)$ & 0.058 \\
\hline $\mathrm{T} 4 \mathrm{t}(\mu \mathrm{g} / \mathrm{ml})$ & 29 & $9.36 \pm 2.7(2.8-17.4)$ & 126 & $9.20 \pm 3.3(0.7-16.6)$ & 0.818 \\
\hline
\end{tabular}

$\mathrm{DE}=$ desviación estándar; $\mathrm{TSH}=$ hormona estimulante tiroidea; $\mathrm{T} 4$ = tiroxina.

* Prueba t de Student.

Tabla 4. Diagnóstico derivados de las pruebas tiroideas en neonatos con síndrome de Down conforme a la historia familiar de enfermedad tiroidea

\begin{tabular}{|c|c|c|c|c|}
\hline \multirow[t]{2}{*}{ Variables } & \multicolumn{2}{|c|}{ Historia familiar de enfermedad tiroidea } & \multicolumn{2}{|c|}{ Razón de momios } \\
\hline & Positiva $n=37(\%)$ & Negativa $n=183(\%)$ & Cruda (IC $95 \%$ ) & Ajustada' (IC $95 \%$ ) \\
\hline Eutiroideo & $23(62.2)$ & $147(80.3)$ & $0.4(0.2-0.8)$ & $0.4(0.1-0.8)$ \\
\hline Hipotiroidismo congénito & $5(13.5)$ & $4(2.2)$ & $6.9(1.8-27.4)$ & $8.3(2.0-34.3)$ \\
\hline Hipotiroidismo subclínico & $9(24.3)$ & $28(15.3)$ & $1.8(0.8-4.2)$ & $1.8(0.8-4.3)$ \\
\hline Síndrome de tiroides enferma & $0(0)$ & $4(2.2)$ & $0.9(0.1-8.7)^{\dagger}$ & $1.3(0.1-11.5)^{\dagger}$ \\
\hline Hipotiroidismo congénito en niñas & $2(5.4)$ & $1(0.5)$ & $10.4(0.9-117.8)$ & $7.1(0.6-86.4)$ \\
\hline Hipotiroidismo congénito en niños & $3(8.1)$ & $3(1.6)$ & $5.3(1.0-27.3)$ & $9.0(1.6-49.6)$ \\
\hline Hipotiroidismo subclínico en niñas & $6(16.2)$ & $10(5.5)$ & $3.3(1.1-9.9)$ & $2.3(0.7-7.3)$ \\
\hline Hipotiroidismo subclínico en niños & $3(8.1)$ & $18(9.8)$ & $0.8(0.2-2.9)$ & $1.3(0.3-5.0)$ \\
\hline
\end{tabular}

IC $95 \%$ = intervalo de confianza del $95 \%$.

*La razón de momios fue ajustada según el sexo de los lactantes.

tEstimado de acuerdo con la corrección $1 / 2$ de Haldane-Anscombe.

crudas y ajustadas se presentan en la Tabla 4. La RM se ajustó para el sexo. Nueve RN con SD en nuestra muestra estudiada tuvieron HC (4.1\%). Todos ellos con una TSH > $10 \mu \mathrm{lU} / \mathrm{mL}$, T4 libre > $0.9 \mathrm{ng} / \mathrm{dL}$ y/o T4 total $<7.2 \mu \mathrm{g} / \mathrm{dL}$. Adicionalmente, 37 presentaron hipertirotropinemia (16.8\%), cuatro síndrome de tiroides enferma (1.8\%) y el resto fue clasificado según el resultado de las PFT como normal (77.3\%). El análisis multivariante mostró que el HC mostró asociación con la HFET en los RN con SD (RMa = 8.3, IC $95 \%$ : 2.0-34.3), particularmente en los RN de sexo masculino (RMa = 9.0, IC 95 \%: 1.6-49.6). La HFET negativa fue un factor protector para $\mathrm{HC}$ en los RN con SD (RMa = 0.4, IC $95 \%$ : 0.1-0.8). Solo uno de los lactantes con $\mathrm{HC}$ nació de una madre hipotiroidea y ninguno de los nacidos con hipertirotropinemia. Para el resto de las variables evaluadas, las RMa no mostraron efecto en los variables estudiadas.

\section{Discusión}

Este estudio empleó PFT venosa por varias razones: a) los RN con SD constituyen una población de alto riesgo para $\mathrm{HC}$, b) el tamizaje para $\mathrm{HC}$ no se practica de forma universal en México, ${ }^{16} \mathrm{c}$ ) existen dificultades en nuestra institución para tener acceso a los resultados del programa de tamizaje de forma rápida y eficiente, y d) los resultados de las PFT están disponibles dentro del mismo día en el laboratorio de nuestro hospital.

Aún así, la frecuencia del $4 \%$ de $\mathrm{HC}$ en nuestra cohorte de RN con SD coincide con los reportes de estudios previos, a pesar de que la mayoría de ellos derivan de programas de tamizaje en $\mathrm{RN}$. $^{1-4,6,17-19}$ Nuestros resultados además soportan que la disfunción tiroidea en los RN con SD es usualmente no severa, incluyendo un $\mathrm{HC}$ leve y más frecuentemente, 
una alta prevalencia de hipertirotropinemia (Tabla 4). Estas particularidades de la función tiroidea en SD se han atribuido a un probable error innato en el metabolismo de las hormonas tiroideas o a la inmadurez del eje hipotálamo-hipofisiario. ${ }^{18}$

Nuestro grupo de estudio fue similar a otros previamente publicados con respecto a las comorbilidades comunes en el SD y también para otros posibles factores de riesgo para $\mathrm{HC}$ aplicables para en población general, ${ }^{4,17}$ excepto por las diferencias en el sexo de los RN (Tabla 2).

Mediante el análisis multivariante se identificó un aumento de riesgo para $\mathrm{HC}$ en RN con SD e HFET y como factor protector, la HFET negativa. El riesgo resulta particularmente alto en aquellos pacientes con SD del sexo masculino (Tabla 4). Dicha asociación no ha sido reportada previamente. Esto sugiere que la historia familiar puede ser considerada como un factor de riesgo para ciertos genotipo(s) susceptible(s)..$^{20} \mathrm{El}$ agrupamiento de $\mathrm{HC}$ en miembros de familiares con SD puede estar explicado mediante los factores genéticos compartidos. Por lo anterior, este estudio sugiere fuertemente que existe un subgrupo de pacientes con $\mathrm{SD}$ con mayor riesgo para $\mathrm{HC}$.

La historia de problemas tiroideos en madres o padres de $\mathrm{RN}$ con $\mathrm{HC}$ se considera un factor de riesgo para $\mathrm{HC}$ en la población general, ${ }^{9}$ evidenciando una clara relación entre la HFET y el HC. En el estudio de Underland et al. ${ }^{21}$, sin embargo, dicha asociación se encontró solo en un análisis bivariante y la HFET positiva fue confinada solo a los padres. Aunque la explicación exacta de la asociación entre la HFET y el HC permanece sin conocerse, la patogénesis autoinmune parece poco probable. En lactantes no malformados, menos del $3 \%$ de los casos con $\mathrm{HC}$ resultan de enfermedad materna autoinmune relacionada con anticuerpos que inhiben la TSH. ${ }^{8,21}$ En nuestro estudio no se evaluó la etiología autoinmune, pero parece también improbable que esta explique la asociación entre la HFET y el HC en nuestros neonatos con SD, ya que solo uno de los lactantes estudiados con $\mathrm{HC}$ tuvo una madre hipotiroidea. En cualquier caso, la agregación familiar de enfermedad tiroidea pone en evidencia una predisposición genética para $\mathrm{HC}$ en $\mathrm{SD}$, pero requerirá obligatoriamente su confirmación posterior, incluyendo su evaluación usando otros marcadores genéticos. El hallazgo de un mayor riesgo de $\mathrm{HC}$ en los varones con SD con HFET positiva no es fácil de explicar (Tabla 4). El sexo femenino ha sido claramente identificado como un factor de riesgo para $\mathrm{HC}$

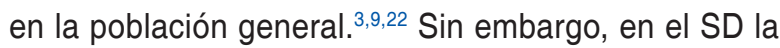

proporción de sexos afectados por $\mathrm{HC}$ no ha sido identificada de manera inequívoca. , $3,5^{3,5}$ Por lo tanto, se requieren futuras investigaciones para explicar la diferencia de sexos observada.

Como una limitación inherente al diseño de casos y controles utilizado, no se puede excluir un sesgo de memoria, debido a que la información fue obtenida mediante entrevistas y autorreportes. No haber determinado la etiología del HC (dishormonogénesis o disgénesis) y no contar con un seguimiento del hipotiroidismo en nuestros pacientes constituyen también debilidades adicionales en nuestro estudio. Además, el número de nuestros pacientes con HFET fue relativamente pequeño, indicando la necesidad de estudios futuros o un metaanálisis de varios reportes publicados.

En conclusión, confirmamos la asociación entre el $\mathrm{HC}$ y la HFET en RN con SD. Los lactantes con SD con HFET tienen un riesgo ocho veces mayor de HC, particularmente los del sexo masculino. La valoración de la HFET mediante un árbol de tres generaciones es una estrategia fácil y accesible para identificar lactantes con mayor riesgo de $\mathrm{HC}$ entre la población de RN con SD y no debe ser subestimada. Sin embargo, los mecanismos causales intrínsecos de esta relación permanecen desconocidos hasta el momento.

\section{Agradecimientos}

Al equipo del CRIAC por su apoyo operativo entusiasta. También al personal de la unidad de citogenética por su asistencia valiosa para el análisis cromosómico.

\section{Conflicto de intereses}

Los autores declaran no tener conflicto de intereses alguno.

\section{Financiamiento}

Los autores no recibieron patrocinio para llevar a cabo este artículo.

\section{Responsabilidades éticas}

Protección de personas y animales. Los autores declaran que para esta investigación no se han realizado experimentos en seres humanos ni en animales. 
Confidencialidad de los datos. Los autores declaran que han seguido los protocolos de su centro de trabajo sobre la publicación de datos de pacientes.

Derecho a la privacidad y consentimiento informado. Los autores han obtenido el consentimiento informado de los pacientes y/o sujetos referidos en el artículo. Este documento obra en poder del autor de correspondencia.

\section{Bibliografía}

1. Bull MJ, Committee on Genetics. Health supervision for children with Down syndrome. Pediatrics. 2011:128:393-406

2. Fort P, Lifshitz F, Bellisario R, Davis J, Lanes R, Pugliese M, et al. Abnormalities of thyroid function in infants with Down syndrome. J Pediatr. 1984:104:545-9.

3. Purdy IB, Singh N, Brown WL, Vangala S, Devaskar UP. Revisiting early hypothyroidism screening in infants with Down syndrome. J Perinatol. 2014;34:936-40.

4. Pierce MJ, LaFranchi SH, Pinter JD. Characterization of thyroid abnormalities in a large cohort of children with Down syndrome. Horm Res Paediatr. 2017;87:170-8.

5. Kariyawasam D, Carré A, Luton D, Polak M. Down syndrome and nonautoimmune hypothyroidisms in neonates and infants. Horm Res Paediatr. 2015;83:126-31

6. van Trotsenburg AS, Kempers MJ, Endert E, Tijssen JG, de Vijlder JJ, Vulsma T. Trisomy 21 causes persistent congenital hypothyroidism presumably of thyroidal origin. Thyroid. 2006;16:671-80.

7. Lavigne J, Sharr C, Elsharkawi I, Ozonoff A, Baumer N, Brasington C, et al. Thyroid dysfunction in patients with Down syndrome: Results from a multi-institutional registry study. Am J Med Genet A. 2017;173:1539-45.

8. Fialkow PJ, Hecht F, Uchida IA, Motulsky AG. Increased frequency of thyroid autoantibodies in mothers of patients with Down's syndrome. Lancet. 1965;2(7418):868-70.

9. Medda E, Olivieri A, Stazi MA, Grandolfo ME, Fazzini C, Baserga M, et al. Risk factors for congenital hypothyroidism: results of a population case-control study (1997-2003). Eur J Endocrinol. 2005;153:765-73.
10. Corona-Rivera JR, Martínez-Macías FJ, Bobadilla-Morales L, Corona-Rivera A, Peña-Padilla C, Ríos-Flores IM, et al. Prevalence and risk factors for Down syndrome: A hospital-based single-center study in Western Mexico. Am J Med Genet A. 2019;179:435-41.

11. Elmlinger MW, Kühnel W, Lambrecht HG, Ranke MB. Reference intervals from birth to adulthood for serum thyroxine (T4), triiodothyronine (T3), free T3, free T4, thyroxine binding globulin (TBG) and thyrotropin (TSH). Clin Chem Lab Med. 2001:39:973-9.

12. Marks AG, LaFranchi SH. Assessing thyroid function in infants and children. En: Brent GA, editor. Thyroid function testing. Nueva York: Springer; 2010. pp. 173-185.

13. Jonklaas J, Bianco AC, Bauer AJ, Burman KD, Cappola AR, Celi FS, et al. Guidelines for the treatment of hypothyroidism: prepared by the American Thyroid Association Task Force on thyroid hormone replacement. Thyroid. 2014;24:1670-751.

14. Bennett RL, French KS, Resta RG, Doyle DL. Standardized human pedigree nomenclature: update and assessment of the recommendations of the National Society of Genetic Counselors. J Genet Couns. 2008;17:424-33.

15. Hinton $C F$, Harris KB, Borgfeld L, Drummond-Borg M, Eaton R, Lorey F, et al. Trends in incidence rates of congenital hypothyroidism related to select demographic factors: data from the United States, California, Massachusetts, New York, and Texas. Pediatrics. 2010;125(Suppl 2):S37-47.

16. Vela-Amieva M, Gamboa-Cardiel S, Pérez-Andrade ME, Ortiz-Cortés J González-Contreras CR, Ortega-Velázquez V. Epidemiology of congenital hypothyroidism in Mexico. Salud Publica Mex. 2004;46:141-8.

17. Jaruratanasirikul S, Patarakijvanich $N$, Patanapisarnsak $C$. The association of congenital hypothyroidism and congenital gastrointestinal anomalies in Down's syndrome infants. J Pediatr Endocrinol Metab. 1998;11:241-6.

18. van Trotsenburg AS, Vulsma T, van Santen HM, Cheung W, de Vijlder JJ. Lower neonatal screening thyroxine concentrations in Down syndrome newborns. J Clin Endocrinol Metab. 2003;88:1512-5.

19. Erlichman I, Mimouni FB, Erlichman M, Schimmel MS. Thyroxine-based screening for congenital hypothyroidism in neonates with Down syndrome. J Pediatr. 2016;173:165-8.

20. Zimmerman R, Pal DK, Tin A, Ahsan H, Greenberg DA. Methods for assessing familial aggregation: family history measures and confounding in the standard cohort, reconstructed cohort and case-control designs. Hum Hered. 2009;68:201-8.

21. Underland L, Kenigsberg L, Derrick KM, Crespi R, Kaushal T, Lam L. Thyroid function testing in neonates with maternal history of disease. Clin Pediatr (Phila). 2018;57:436-41.

22. Waller DK, Anderson JL, Lorey F, Cunningham GC. Risk factors for congenital hypothyroidism: an investigation of infant's birth weight, ethnicity, and gender in California 1990-1998. Teratology. 2000;62:36-41. 